\title{
Efeito do substrato, cor e tamanho de sementes na germinação e vigor de Melanoxylon brauna
} \author{
Guimarães², Glauciana da Mata Ataíde ${ }^{2}$, Daniela de Pádua Barros², Márcio Dias Pereira ${ }^{4}$ \\ ${ }^{1}$ Universidade Federal de Santa Catarina, Rod. Ulisses Gaboardi, Km 3, CP 101, CEP 89520-000, Curitibanos, SC, Brasil \\ 2Universidade Federal de Viçosa, Av. Peter Henry Rolfs, s/nº, CEP 36570-000, Viçosa, MG, Brasil \\ ${ }^{3}$ Instituto Nacional de Pesquisas da Amazônia, Av. Efigênio Sales, CP 476, CEP 69011-970, Manaus, AM, Brasil \\ ${ }^{4}$ Universidade Federal de Rio Grande do Norte, Av. Senador Salgado Filho, 3000, CEP 59078-970, Natal, RN, Brasil
}

Andressa Vasconcelos Flores', Eduardo Euclydes de Lima e Borges², José Francisco de Carvalho Gonçalves ${ }^{3}$, Valéria Monteze

\section{"Autor correspondente:}

andressafloressm@yahoo.com.br

Termos para indexação:

Fisiologia

Viabilidade

Propagação

Morfologia

Index terms:

Physiology

Viability

Propagation

Morphology

Histórico do artigo:

Recebido em 26/06/2013

Aprovado em 15/04/2014

Publicado em 07/07/2014

doi: 10.4336/2014.pfb.34.78.558
Resumo - O objetivo deste trabalho foi investigar o efeito do substrato, da cor e do tamanho das sementes sobre a germinação e vigor de Melanoxylon brauna. Para tanto foram realizados dois ensaios. No primeiro, as sementes foram classificadas quanto à cor (claras e escuras) e ao tamanho (pequenas e grandes). No segundo ensaio foi investigada a influência dos substratos sobre areia, entre areia, sobre papel, entre papel e rolos de papel, tipo germitest. Em ambos os ensaios, as sementes foram mantidas em condições controladas (luz e temperatura) durante 10 dias. Foi calculado o percentual de germinação e o índice de velocidade de germinação (IVG). Além disso, foi determinado o peso de 1.000 sementes e a germinação da espécie foi caracterizada mediante acompanhamento visual até a formação da plântula. O peso de 1.000 sementes de $M$. brauna foi de 133,43 g, em média. A protrusão da raiz principal ocorreu em torno de 72 horas após início da embebição, e a germinação observada foi do tipo fanerocotiledonar epígea. Sementes pequenas e claras apresentaram germinação e IVG inferiores às demais. As sementes grandes, independentes da cor, e as pequenas escuras tiveram os maiores IVG e porcentagem de germinação. O substrato mais indicado para a realização de testes de germinação de Melanoxylon brauna foi o rolo de papel.

\section{Effect of substrate, color and size on germination and vigor of Melanoxylon brauna seeds}

\begin{abstract}
The objective of this work was to investigate the effect of substrate, color and size on the germination and vigor of Melanoxylon brauna seeds. It was performed two essays. In the first, the seeds were classified by color (light and dark) and size (small and large). In the second essay, it was investigated the influence of substrates on sand, between sand, above paper, between paper and in paper rolls, type germitest. In both essays, the seeds were maintained in controlled conditions (light and temperature) during 10 days. The percentage of germination and germination speed index (GSI) were calculated. Furthermore, it was determined the weight of 1,000 seeds and the germination of the species was characterized by observation until the seedling formation. The weight of 1,000 seeds of M. brauna was $133.43 \mathrm{~g}$, in average. The root protrusion occurred around 72 hours after the beginning of imbibition, and the germination was as epigel phanerocotyledonar. Small and light color seeds presented percentage of germination and GSI lower than others. The large seeds, independent of color, and small dark seeds presented the higher GSI and percentage of germination. Paper roll is the substrate indicated to conduct germination tests of Melanoxylon brauna.
\end{abstract}




\section{Introdução}

O conhecimento a respeito das características inerentes às sementes como a forma, o tamanho e a cor, além do substrato mais adequado para o melhor desempenho do processo germinativo constituem importantes fatores na propagação de diferentes espécies vegetais. As influências de cada característica e do substrato possuem graus variáveis de importância de acordo com a espécie.

A cor do tegumento das sementes tem influência sobre a sua qualidade fisiológica e está associada ao grau de maturação, sendo as sementes de coloração mais clara as mais imaturas (Lucena et al., 2008), à semelhança de sementes de Pothomorpha umbellata em que MaiaAlmeida et al. (2011) concluíram que as sementes pretas possuíam maiores porcentagem e velocidade de germinação, sincronia e densidade. Frutos de Mimosa caesalpinifolia apresentaram $81 \%$ de germinação quando na cor marrom-opaco, típica de frutos maduros (Freitas et al., 2013). Da mesma forma, sementes de Jacaranda decurrens, subsp. symmetrifoliolata, na cor castanho-claro, portanto mais imatura, germinaram mais rápido, embora não tenha havido diferença significativa das cores na germinação (Sangalli et al., 2004).

O tamanho das sementes é uma das características que apresenta grande variabilidade, sendo definido pelo comprimento, largura e espessura. As plantas produzem sementes de tamanhos completamente desuniformes pelas variações na disponibilidade de recursos nutricionais durante o desenvolvimento dos frutos (Winn, 1991). O tamanho pode ser um indicativo da qualidade fisiológica das sementes para muitas espécies e, geralmente, nesses casos, as pequenas apresentam menores valores de germinação e vigor quando comparadas com as de tamanho médio e grande (Biruel et al., 2010). Sementes maiores originam plântulas mais vigorosas, com tamanho e massa maior que plântulas provenientes de sementes menores (Vanzolini \& Nakagawa, 2007).

O substrato é um dos fatores que afetam o comportamento das sementes durante a condução dos testes de germinação (Alves et al., 2002; Cetnarski Filho \& Carvalho, 2009). Para a escolha do substrato de germinação devem ser observadas algumas características do material a ser utilizado, tais como a composição, a capacidade de retenção de água, a estrutura porosa, o $\mathrm{pH}$, a pureza microbiológica, a resistência, a toxidez, o tamanho e a textura (Brasil, 2009). O fato é que no substrato as sementes são condicionadas para germinarem, e este tem a função de manter as condições adequadas para que o processo germinativo ocorra (Figliolia et al., 1993). Os substratos mais utilizados em testes de germinação em diferentes laboratórios e pesquisas são o papel filtro, o papel toalha, a areia, a vermiculita, a terra, o papel mataborrão e o carvão (Oliveira et al., 1996).

Melanoxylon brauna na nomenclatura popular é conhecida como braúna, braúna preta, graúna, maria-preta ou rabo de macaco (Lorenzi, 2002). Ocorre naturalmente no sul da Bahia, São Paulo, Minas Gerais, Espírito Santo, Pará, Rio de Janeiro e Sergipe. É classificada como semidecídua, heliófila, com ocorrência também na floresta pluvial da encosta atlântica (Almeida et al., 1998; Lorenzi, 2002). Sua madeira é muito pesada, apresentando densidade de $1,05 \mathrm{~g} \mathrm{~cm}^{-3}$, compacta, muito dura ao corte e de grande durabilidade, mesmo quando em ambientes adversos, sendo própria para obras externas e hidráulicas, dentre outros. É uma espécie potencial para reflorestamentos (Lorenzi, 2002). Devido à exploração de sua madeira e à falta de plantios, a espécie está presente na "lista oficial de flora ameaçada de extinção" estabelecida pela Instrução Normativa $\mathrm{N}^{\circ}$ 6, de 23 de setembro de 2008 (Brasil, 2008).

As sementes de $M$. brauna constituem a via mais utilizada na propagação desta espécie. $\mathrm{O}$ efeito da cor do tegumento, do tamanho da semente e do substrato de germinação ainda não foi pesquisado para a espécie. $M$. brauna pertencente à família Fabaceae.

Considerando-se a necessidade de melhor conhecer a influência de fatores intrínsecos das sementes de braúna, bem como o substrato que proporcione melhor germinação, o objetivo deste trabalho foi avaliar o efeito da cor, tamanho e substrato na germinação e vigor de sementes de Melanoxylon brauna.

\section{Material e métodos}

\section{Material vegetal e beneficiamento}

As sementes de $M$. brauna foram colhidas em 10 árvores-matrizes na cidade de Leopoldina, MG. Os trabalhos foram conduzidos no Laboratório de Análise de Sementes Florestais do Departamento de Engenharia Florestal da Universidade Federal de Viçosa. Após a 
colheita, a secagem dos frutos foi realizada ao sol até sua abertura e as sementes, contendo aproximadamente $13 \%$ de teor de água, determinado conforme Brasil (2009). As sementes foram armazenadas logo após a colheita/beneficiamento, em tambores de fibra a $5{ }^{\circ} \mathrm{C} \mathrm{e}$ $60 \%$ de umidade relativa. O beneficiamento consistiu em eliminar as sementes imaturas, deterioradas ou danificadas.

\section{Determinação do peso de $\mathbf{1 . 0 0 0}$ sementes}

O peso de 1.000 sementes foi calculado para o lote de acordo com o procedimento descrito por Brasil (2009).

\section{Processo germinativo}

Anteriormente ao teste de germinação, as sementes foram imersas em solução de fungicida CAPTAN 0,5\% por dois minutos e colocadas em placas de petri com $9,0 \mathrm{~cm}$ de diâmetro sobre duas folhas de papel germistet (Experimento I) ou diferentes substratos (Experimento II). Em ambos, os substratos foram umedecidos com água destilada. Os experimentos foram realizados em câmara tipo BOD, com temperatura constante de $25{ }^{\circ} \mathrm{C}$, sob luz contínua, proporcionada por quatro lâmpadas de $20 \mathrm{~W}$, tipo luz do dia, por 10 dias. Foram consideradas germinadas as sementes que emitiram raiz primária $(2 \mathrm{~mm})$. A reidratação foi realizada sempre que necessário. O número de sementes germinadas foi avaliado diariamente, sendo calculados a porcentagem de germinação e o índice de velocidade de germinação - IVG (Maguire, 1962).

Durante o teste de germinação, no substrato rolo de papel germitest, selecionaram-se cinco sementes (a mais central em cada repetição) para avaliação visual do processo germinativo, observando-se as alterações morfológicas ao longo de oito dias.

\section{Ensaio I}

Para avaliar o efeito da cor e do tamanho, as sementes foram classificadas visualmente em claras e escuras e em pequenas $(5,05( \pm 0,41) \times 11,59( \pm 0,99) \mathrm{mm}) \mathrm{e}$ grandes $(6,00( \pm 0,64) \times 13,33( \pm 0,81) \mathrm{mm})$ (Figura 1). Em seguida, o comprimento e a largura foram medidos para a caracterização das classes.

\section{Ensaio II}

Foram testados os substratos sobre areia (SA), entre areia (EA), sobre papel (SP), entre papel (EP) e rolo de papel (RP). Nos tratamentos com papel, o tipo utilizado foi o germitest.

\section{Delineamento experimental}

O delineamento experimental utilizado no experimento I foi o inteiramente casualizado, em esquema fatorial $2 \mathrm{x}$ 2 (duas cores e dois tamanhos) e inteiramente casualizado com cinco tratamentos no experimento II, ambos com cinco repetições de 20 sementes. A análise estatística foi realizada por meio de análise de variância e as médias foram comparadas pelo teste de Tukey a 5\% de probabilidade, utilizando-se o programa SAS.

\section{Resultados e discussão}

As sementes apresentaram teor de água de $13 \%$ e o peso médio foi de 133,43 g para 1.000 sementes, apresentando coeficiente de variação de $1,5 \%$. Um quilograma de sementes de Melanoxylon brauna possui em média 7.494 sementes, valor que diverge dos valores encontrados nas Instruções para Análise de Sementes de Espécies Florestais (Brasil, 2013) que é de 10.600 a 30.000 sementes por quilograma. Lorenzi (2002) também menciona valores próximos a 30.000 sementes por quilograma. Para Cetnarski Filho \& Nogueira (2005), essa variação pode ocorrer devido a fatores como o local de coleta, a idade das matrizes ou o teor de água das sementes.
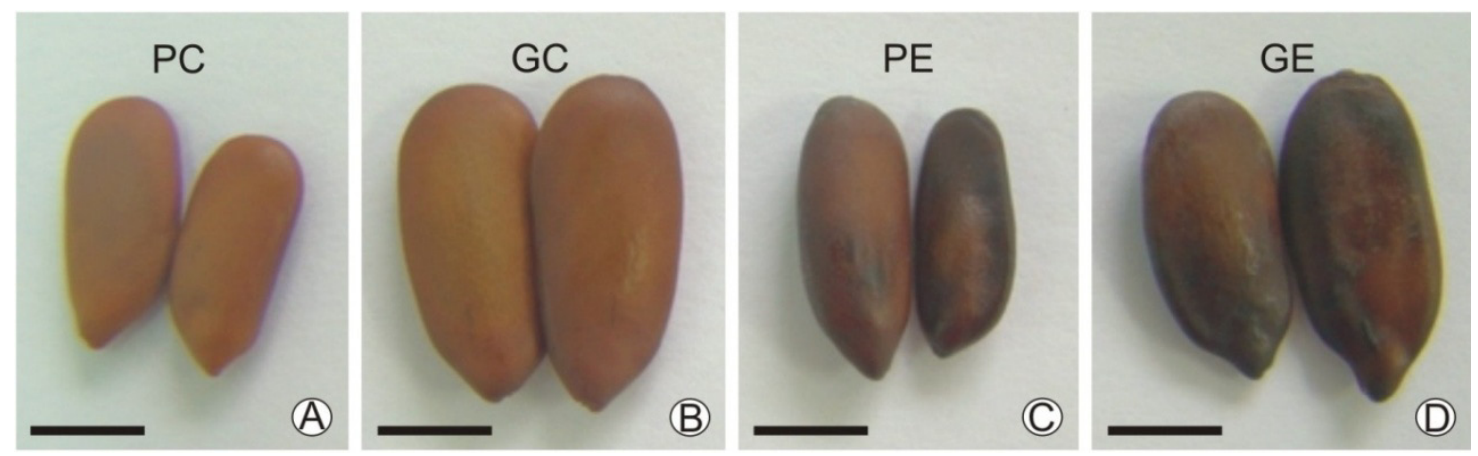

Figura 1. Sementes de Melanoxylon brauna classificadas em relação à cor e ao tamanho. A) PC = pequenas e claras; $\mathrm{B}) \mathrm{GC}=$ grandes e claras; C) $\mathrm{PE}=$ pequenas e escuras; e D) $\mathrm{GE}=$ grandes e escuras. Barra $=5 \mathrm{~mm}$. 
As etapas do processo de germinação das sementes de M. brauna em função do tempo podem ser observadas na Figura 2. A primeira alteração observada foi o aumento significativo do tamanho das sementes. Em aproximadamente $72 \mathrm{~h}$ ocorre a protrusão da raiz principal. No decorrer do crescimento e desenvolvimento da plântula observa-se a diferenciação entre a raiz principal e o hipocótilo, com a concomitante liberação dos cotilédones, por volta do sexto dia. Em seguida, a partir do oitavo dia, começam a surgir os eofilos. A germinação das sementes de M. brauna é fanerocotiledonar epígea.

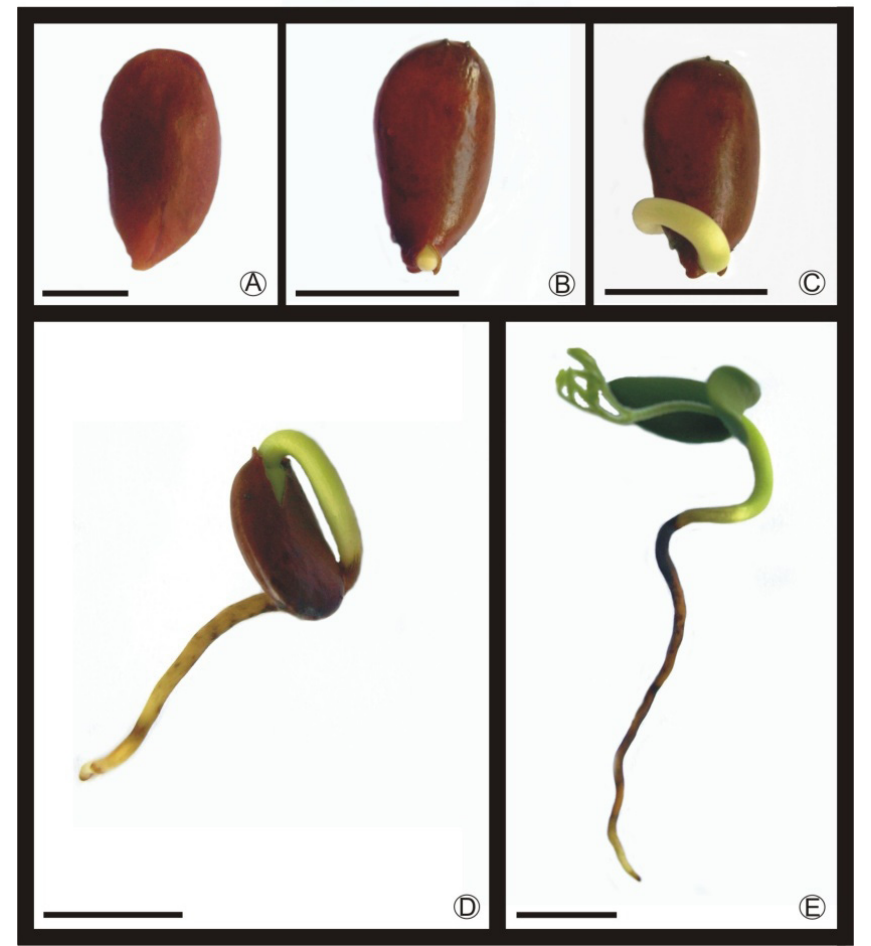

Figura 2. Aspectos da germinação da semente de Melanoxylon brauna e da formação da plântula, sob temperatura de $25^{\circ} \mathrm{C}$ em câmara tipo BOD. Onde: A) zero hora de semeadura; B) 72 h após a semeadura (protrusão da raiz principal); C) 96 h após a semeadura; D) 120 h após a semeadura; E) plântula com 192 h após a semeadura. Figura 2A, Barra = 5 mm, e Figuras 2B, $2 \mathrm{C}, 2 \mathrm{D}$ e $2 \mathrm{E}$, Barra $=10 \mathrm{~mm}$.

As características cor e tamanho apresentaram interação significativa para o percentual de germinação e IVG (Tabela 1). Sementes pequenas e claras apresentaram 69\% de germinação e IVG de 2,44, valores significativamente inferiores quando comparado com as sementes pequenas de coloração escuras e as grandes de coloração clara. As pequenas escuras não diferiram na porcentagem de germinação e no IVG das grandes escuras, que, por sua vez, não diferiram das grandes claras. Mesmo considerando que as sementes pequenas e claras diminuem a qualidade fisiológica do lote, elas apresentam percentual de germinação relevante de $69 \%$, indicando que a coloração pode ser um indicador morfológico de estágio de maturação para as sementes desta espécie.
Tabela 1. Germinação (\%) e índice de velocidade de germinação (IVG) de sementes de Melanoxylon brauna para diferentes cores e tamanhos.

\begin{tabular}{|c|c|c|c|c|}
\hline \multirow{3}{*}{ Cor } & \multicolumn{4}{|c|}{ Tamanho } \\
\hline & \multicolumn{2}{|c|}{ G (\%) } & \multicolumn{2}{|c|}{ IVG } \\
\hline & Pequena & Grande & Pequena & Grande \\
\hline Clara & $69,00 \mathrm{Bb}^{*}$ & $96,00 \mathrm{Aa}$ & $2,44 \mathrm{Ab}$ & 3,34 Aa \\
\hline Escura & $93,00 \mathrm{Aa}$ & $91,00 \mathrm{Aa}$ & $3,46 \mathrm{Ba}$ & 3,33 Aa \\
\hline
\end{tabular}

*Médias seguidas pela mesma letra maiúscula nas colunas (cor) e minúscula nas linhas (tamanho) não diferem entre si, pelo teste de Tukey $(\mathrm{p}>0,05)$. 
Lucena et al. (2008) classificaram as sementes de Ricinus communis em diferentes cores e concluíram que quanto mais clara, menor o vigor e a porcentagem de emergência. Os autores afirmam que a cor do tegumento está associada ao grau de maturação, sendo que sementes mais claras são mais imaturas. Este fenômeno também foi verificado nas sementes de braúna.

O tamanho das sementes também foi estudado para as espécies Simmondsia chinensis (Figueiredo, 1989), Mimosa caesalpiniaefolia (Alves et al., 2005), Syzygium malaccense (Costa et al., 2006), Schizolobium amazonicum (Ghisolfi et al., 2006), Hyptis suaveolens (Mandal et al., 2008) e Caesalpinia leiostachya (Biruel et al., 2010), verificando-se que, em geral, sementes grandes apresentaram maiores porcentagem de germinação e vigor.

Não foi verificado efeito significativo do substrato na porcentagem de germinação (Tabela 2). Comportamento semelhante foi obtido em estudo com diferentes substratos (vermiculita, entre areia, sobre papel, sobre areia e rolo de papel) em sementes de Solanum sessiflorum (Lopes \& Pereira, 2005). Entretanto, foram observadas diferenças significativas entre os substratos testados para Torresia acreana (Albrecht et al., 1986), Cedrela odorata (Andrade \& Pereira, 1994), Sebastiana commersoniana (Santos \& Aguiar, 2000), Mimosa caesalpiniaefolia (Alves et al., 2002), Cnidosculus phyllacanthus (Silva \& Aguiar, 2004), Eucalyptus dunni (Cetnarski Filho \& Carvalho, 2009) e Eugenia uniflora (Sena et al., 2010).

Tabela 2. Germinação (\%) e índice de velocidade de germinação (IVG) para sementes de Melanoxylon brauna em diferentes substratos de germinação.

\begin{tabular}{lcl}
\hline \multirow{2}{*}{ Substratos } & \multicolumn{2}{c}{ Variáveis } \\
\cline { 2 - 3 } & Germinação (\%) & IVG \\
\hline Sobre areia (SA) & $79,0 \mathrm{a}^{*}$ & $2,55 \mathrm{c}^{*}$ \\
Entre areia (EA) & $89,0 \mathrm{a}$ & $3,79 \mathrm{ab}$ \\
Sobre papel (SP) & $88,0 \mathrm{a}$ & $2,62 \mathrm{c}$ \\
Entre papel (EP) & $92,0 \mathrm{a}$ & $3,43 \mathrm{~b}$ \\
Rolo de papel (RP) & $94,0 \mathrm{a}$ & $4,52 \mathrm{a}$ \\
\hline
\end{tabular}

*Médias seguidas pela mesma letra na coluna não diferem entre si, pelo teste de Tukey $(\mathrm{p}>0,05)$

Os substratos, rolo de papel e entre areia proporcionaram maiores valores de IVG, em relação aos demais, mas não houve diferença entre os mesmos. Por outro lado, os tratamentos sobre papel e sobre areia não diferiram entre si e tiveram os valores significativamente menores em relação aos demais. Isto sugere que a área de contato do substrato umedecido com a semente é muito importante, podendo não ser crítica para a germinação total, mas afeta a velocidade de germinação. Os resultados observados estão de acordo com Carneiro \& Guedes (1992), que verificaram que quanto maior é o contato das sementes de Stevia rebaudiana com o substrato, maior a percentagem de germinação e menor o tempo necessário para alcançála. Dessa forma, devido à praticidade de utilizar o rolo de papel como substrato em laboratório, a baixa contaminação por microorganismos e a facilidade de avaliação das sementes, este é indicado para a condução de testes de germinação de sementes de M. brauna. Estes resultados estão de acordo com as Instruções para Análise de Sementes de Espécies Florestais (Brasil, 2013) que indica como substratos para realização de testes de germinação para a espécie os substratos rolo de papel e sobre vermiculita.

\section{Conclusões}

Sementes pequenas e claras apresentam germinação e índice de velocidade de germinação inferiores às demais.

As sementes grandes, independentes da cor, e as pequenas escuras apresentaram o maior índice de velocidade de germinação e a maior porcentagem de germinação.

O substrato mais indicado para a realização de testes de germinação de M. brauna é o rolo de papel.

\section{Agradecimentos}

Ao CNPq.

\section{Referências}

ALBRECHT, J. M. F.; ALBUQUERQUE, M. C. de L. F.; SILVA, V. S. de M. e. Influência da temperatura e do tipo de substrato na germinação de sementes de cerejeira. Revista Brasileira de Sementes, Pelotas, v. 8, n. 1, p. 49-55, 1986.

ALMEIDA, S. P.; PROENÇA, C. E. B.; SANO, S. M.; RIBEIRO, J. F. Cerrado: espécies vegetais úteis. Planaltina: EMBRAPA-CPAC, 1998. 464 p.

ALVES, E. U.; PAULA, R. C. de; OLIVEIRA, A. P.; BRUNO, R. L. A.; DINIZ, A. A. Germinação de sementes de Mimosa caesalpiniaefolia Benth em diferentes substratos e temperatura. Revista Brasileira de Sementes, Pelotas, v. 24, n. 1, p. 169-178, 2002. 
ALVES, E. U.; BRUNO, R. de L. A.; OLIVEIRA, A. P. de; ALVES, A. U.; ALVES, A. U.; PAULA, R. C. de Influência do tamanho e da procedência de sementes de Mimosa caesalpiniaefolia Benth. sobre a germinação e vigor. Revista Árvore, Viçosa, MG, v. 29, n. 6, p. 877-885, 2005.

ANDRADE, A. C. S. de; PEREIRA, T. S. Efeito do substrato e da temperatura na germinação e no vigor de sementes de cedro Cedrela odorata L. (Meliaceae). Revista Brasileira de Sementes, Pelotas, v. 16, p. 34-40, 1994.

BIRUEL, R. P.; PAULA, R. C. de; AGUIAR, I. B. de. Germinação de sementes de Caesalpinia leiostachya (Benth) Ducke (pau-ferro) classificadas pelo tamanho e pela forma. Revista Árvore, Viçosa, MG, v. 34, n. 2, p. 197-204, 2010.

BRASIL. Ministério da Agricultura, Pecuária e Abastecimento. Secretaria de Defesa Agropecuária. Regras para análise de sementes. Brasília, DF: Mapa/ACS, 2009. 395 p.

BRASIL. Ministério da Agricultura, Pecuária e Abastecimento. Secretaria de Defesa Agropecuária. Instruções para análise de sementes de espécies florestais. Brasília, DF: Mapa/ACS, 2013. $98 \mathrm{p}$.

BRASIL. Ministério do Meio Ambiente. Instrução normativa n. 6 de 23 de setembro de 2008. Lista as espécies da flora brasileira ameaçadas de extinção e com deficiência de dados. Disponível em: <http://www.mma.gov.br/estruturas/ascom_boletins/_ arquivos/83_19092008034949.pdf>. Acesso em: 20 jan. 2011. Publicado originalmente no Diário Oficial [da] República Federativa do Brasil, Brasília, DF, n. 185, seção 1, p. 75-83.

CARNEIRO, J. W. P.; GUEDES, T. A. Influência do contato das sementes de stevia (Stevia rebaudiana (Bert.) Bertoni) no substrato avaliada pela função de Weibull. Revista Brasileira de Sementes, Pelotas, v. 14, n. 1, p. 65-68, 1992.

CETNARSKI FILHO, R.; CARVALHO, R. I. N. de Massa da amostra, substrato e temperatura para teste de germinação de sementes de Eucalyptus dunni Maiden. Ciência Florestal, Santa Maria, RS, v. 19, n. 3, p. 257-265, 2009.

CETNARSKI FILHO, R.; NOGUEIRA, A. C. Influência da temperatura na germinação de diásporos de Ocotea odorifera (Vellozo) Rohwer (canela-sassafrás). Ciência Florestal, Santa Maria, RS, v. 15, n. 2, p. 191-198, 2005.

COSTA, R. S.; OLIVEIRA, I. V. de M.; MÔRO, F. V.; MARTINS, A. B. G. Aspectos morfológicos e influência do tamanho da semente na germinação do jambo-vermelho. Revista Brasileira de Fruticultura, Jaboticabal, v. 28, n. 1, p. 117-120, 2006.

FIGLIOLIA, M. B.; OLIVEIRA, E. C.; PIÑA-RODRIGUES, F. C. M. Análise de sementes. In: AGUIAR, I. B.; PIÑA-RODRIGUES, F. C. M.; FIGLIOLIA, M. B. (Coord.). Sementes florestais tropicais. Brasília, DF: ABRATES, 1993. p. 137-174.

FIGUEIREDO, S. F. L. Germinação de sementes de jojoba ((Simmondsia chinensis (Link) Schneider). Revista Brasileira de Fisiologia Vegetal, Campinas, v. 1, n. 1, p. 99-107, 1989.
FREITAS, T. P.; FREITAS, T. A. S.; CAMPOS, B. M.; FONSECA, M. D. S; MENDONÇA, A. V. R. Morfologia e caracterização da germinação em função da posição das sementes no fruto de sabiá. Scientia Plena, v. 9, n. 3, p. 1-9, 2013.

GHISOLFI, E. M.; EFFGEN, E. M.; MENDONÇA, A. R. da; NAPPO, M. E.; SILVA, A. G. da. Influência do tamanho da sementes e tipo de recipiente na germinação de Schizolobium amazonicum (Herb) Ducke. Revista Científica Eletrônica de Agronomia, Garça, n. 9, 2006.

LOPES, J. C.; PEREIRA, M. D. Germinação de sementes de cubiu em diferentes substratos e temperaturas. Revista Brasileira de Sementes, Pelotas, v. 27, n. 2, p. 146-150, 2005.

LORENZI, H. Árvores brasileiras: manual de identificação e cultivo de plantas arbóreas do Brasil. Nova Odessa: Instituto Plantarum, 2002. v. 1.368 p.

LUCENA, A. M. A. de; SEVERINO, L. S.; BELTRÃO, N. E. de M.; SOFIATTI, V.; MEDEIROS, K. A. L.; OLIVEIRA, M. I. P. de; BORTOLUZI, C. R. D. Influência do estádio de maturação da semente e da profundidade de semeio I: emergência das plântulas e área foliar dos cotilédones. In: CONGRESSO BRASILEIRO DE MAMONA, 3., 2008, Salvador. Energia e ricinoquímica: resumos. Salvador: SEAGRI: Embrapa Algodão, 2008. p. 99.

MAGUIRE, J. D. Speed of germination and in selection and evaluation from seeding emergence and vigor. Crop Science, Madison, v. 2, n. 2, p. 176-177, 1962.

MAIA-ALMEIDA, C. I.; CAVARIANI, C.; OLIVEIRA, P. F.; MING, L. C.; MATTANA, R. S.; LIMA, L. P. Comportamento germinativo de sementes de diferentes cores de Pariparoba (Pothomorpha umbellata (L.) Mig). Revista Brasileira de Plantas Medicinais, Botucatu, v. 13, n. 1, p. 116-120, 2011.

MANDAL, S. M.; CHAKRABORTY, D.; GUPTA, K. Seed size variation: influence on germination and subsequent seedling performance in Hyptis suaveolens (Lamiaceae). Research Journal of Seed Science, New York, v. 1, n. 1, p. 26-33, 2008.

OLIVEIRA, E. C.; PIÑA-RODRIGUES, F. C.; FIGLIOLIA, M. B. Propostas para a padronização de metodologias em análise de sementes florestais. Revista Brasileira de Sementes, Pelotas, v. 11, n. 1-3, p. 1-42, 1996.

SANGALli, A.; SCALON, S. P. Q.; VIEIRA, M. C. Cor, temperratura e pré-eembebição na germinação de sementes de carobinha (Jacaranda decurrens subsp symmetrifoliolata Farias e Proença) Bignoniaceae. Revista Brasileira de Plantas Medicinais, Botucatu, v. 7, n. 1, p. 79-85, 2004.

SANTOS, S. R. G. dos; AGUIAR, I. B. de Germinação de sementes de branquilho (Sebastiania commersoniana (Baill.) Smith \& Downs) em função do substrato e do regime de temperatura. Revista Brasileira de Sementes, Pelotas, v. 22, n. 1, p. 120-126, 2000.

SENA, L. H. de M.; MATOS, V. P.; SALES, A. G. de F. A.; FERREIRA, E. G. B. de S.; PACHECO, M. V. Qualidade fisiológica de sementes de pitangueira submetidas a diferentes procedimentos de secagem e substratos - Parte 2. Revista Brasileira de Engenharia Agrícola e Ambiental, Campina Grande, v. 14, n. 4, p. 412-417, 2010. 
SILVA, L. M. de M.; AGUIAR, I. B. Efeito dos substratos e temperaturas na germinação de sementes de Cnidosculus phyllacanthus Pax\& K. Hoffm. (faveleira). Revista Brasileira de Sementes, Pelotas, v. 26, n. 1, p. 9-14, 2004.

VANZOLINI, S.; NAKAGAWA, J. Testes de vigor baseados no desempenho de plântulas. Informativo ABRATES, Curitiba, v. 17, n. 1-3, p. 76-83, 2007.
WINN, A. A. Proximate and ultimate sources of within individual variation in seed mass in Prunella vulgaris (Lamiaceae). Australian Journal of Botany, Melbourne, v. 78, p. 838-844, 1991. 
